\title{
LINEARIZED PLANING-SURFACE THEORY WITH SURFACE TENSION. PART I: SMOOTH DETACHMENT
}

\author{
E. O. TUCK
}

(Received 1 May 1980)

(Revised 12 November 1980)

\begin{abstract}
In the absence of surface tension, the problem of determining a travelling surface pressure distribution that displaces a portion of the free surface in a prescribed manner has been solved by several authors, and this "planing-surface" problem is reasonably well understood. The effect of inclusion of surface tension is to change, in a dramatic way, the singularity in the integral equation that describes the problem. It is now necessary in general to allow for isolated impulsive pressure, as well as a smooth distribution over the wetted length. Such pressure points generate jump discontinuities in free-surface slope. Numerical results are obtained here for a class of problems in which there is a single impulse located at the leading edge of the planing surface and detachment with continuous slope at the trailing edge. These results do not appear to approach the classical results in the limit as the surface tension approaches zero, a paradox that is resolved in Part II, which follows.
\end{abstract}

\section{Introduction}

This paper addresses itself primarily to a mathematical question arising in the problem of two-dimensional irrotational flow, under a prescribed finite-length fixed surface of small draft, and a free surface with gravity and surface tension. The problem is thus relevant to the practical problem of planing of a highaspect-ratio vessel. In the limit as the penetration of the vessel beneath the free surface tends to zero, the disturbance it causes to the stream vanishes and hence, to leading order of smallness of draft, we may expect to be able to linearize the free-surface boundary condition.

(1) Copyright Australian Mathematical Society 1982 
In the absence of surface tension, this problem has long been considered solved, and is reviewed by Wehausen and Laitone in [7], page 587. The standard procedure is to seek a fictitious travelling pressure distribution, $P(x)$, to replace the planing surface. If such a $P(x)$ is known, it is straightforward to write down the linearized free-surface displacement it generates. All that is necessary then is to equate the generated (small) free-surface displacement with the given (small) displacement of the planing vessel, over the finite segment corresponding to that vessel. In practice, this leads to a singular integral equation of the first kind, to determine $P(x)$, that is readily solvable with modern computers. The computed results appear to be quite reasonable, and to predict pressure distributions and lift in accordance with measurements.

There are a few aspects of this classical problem that are still not fully understood, however, even without surface tension. The integral equation is singular and in principle allows infinitely many solutions. However, a unique solution for $P(x)$ is obtainable if we insist that the free-surface slope agrees with the planing-surface slope where they join at the trailing edge; thus this is equivalent to the Kutta condition of aerodynamics.

Even though the resulting $P(x)$ is uniquely determined for a given input hull, there is still a certain amount of confusion, reflected in the literature on the problem, regarding the connection to the original input surface. The fact is that in general there is no solution at fixed wetted length, for a given planing-surface shape over that length! If we do insist on prescribing the wetted length, the hydrodynamics will not accept just any input shape; it insists on its own degree of freedom, equivalent to a vertical up or down shift of the planing surface as a rigid body. In practice, this is a reflection of the fact that, for a given planing-surface shape of small draft that is not allowed to move up or down, the hydrodynamics must be allowed to determine how much of that surface is wetted at speed, by moving the leading edge forward or back an appropriate amount.

Those authors (for example, Squire [6] and Oertel [5]) who have concerned themselves with this aspect of the planing problem, have found it convenient to dispense a posteriori with the idea of fixing wetted length. That is, although it is convenient (if not almost essential) to fix the wetted length in order to perform the computations, once $P(x)$ is determined it is better to re-plot the results in such a way that the wetted length itself is an output quantity. Thus the conventional wetted-length-based Froude number is a rather inappropriate measure of speed for planing vessels, and other more-practical choices suit the final output better. Oertel [5] also concerns himself with some additional quite difficult questions, that occur for planing surfaces without sharp trailing edges or transoms; here, yet another uncertainty enters, because the water must now be allowed to choose not only the leading but also the trailing-edge location. 
These difficulties are mentioned now, only to add weight to the additional difficulties, only partly-resolved here, that arise when surface tension is significant. These are difficulties associated with the fact that surface tension smooths the free-surface displacement generated by non-smooth pressure distributions.

For example, suppose we consider $P(x)=\delta(x)$, that is, a travelling point impulse of pressure. Then (Lamb [3], page 403) the free-surface displacement at $\boldsymbol{x}=\mathbf{0}$ (that is, immediately beneath the point pressure singularity) is unbounded in the absence of surface tension, specifically tending to infinitely like $\log |x|$ as $x \rightarrow 0$. This logarithmic singularity is the fundamental singularity of the integral equation described earlier.

However, when surface tension, however small, is present, such a $\delta$-function of imposed pressure no longer generates an infinite free-surface displacement. Indeed (Lamb [3], page 465) the resulting free-surface displacement is everywhere continuous. There is, however, a discontinuity in the slope of the free surface at the singular point $x=0$, of a magnitude proportional to the strength of the $\delta$-function.

At first sight, this is good news. Surely, such a more-gentle disturbance, created by a very non-gentle disturber, is helpful in solving the problem? Unfortunately, it is not. Indeed, the singularity in the surface-tension-less case is of great benefit, in producing a solvable integral equation. Non-singular firstkind integral equations are notoriously hard to solve, and the planing-surface integral equation is almost non-singular, when surface tension is present.

A non-existence result, very much more dramatic than the one described above, now occurs. Specifically, at fixed wetted length, no smooth solution for $P(x)$ exists unless we allow the hydrodynamics not only to move the input hull up and down, but also to rotate or pitch it. But this is quite unacceptable, since, for example, it implies that there is no solution at all for a flat plate at a given (small but non-zero) angle or attack. The hydrodynamics will simply shift and rotate it, until there is no displacement and no disturbance to the stream at all.

How do we escape from this dilemma? The only way out is to abandon the requirement that $P(x)$ be smooth. Once this is accepted, its reasonableness becomes apparent. Since a pressure distribution as singular as $P(x)=\delta(x)$ produces a quite-acceptable (even continuous!) free-surface displacement, why should not such singularities be present in the generating (fictitious) pressure distribution? Indeed, we should anticipate presence of such a $\delta$-function in $P(x)$ whenever there is a slope discontinuity in the body and/or free surface.

The external pressure distribution, including the above-mentioned $\delta$-function singularity, is in principle only a mathematical artifice, arranged so as to generate the given planing surface. However, the actual hydrodynamic pressure distribution in the flow immediately beneath the free surface or the planing surface may be obtained directly from this fictitious external pressure, the 
difference being simply proportional to the surface curvature in each case. No singularities of $\delta$-function type ocur in the interior pressure, the $\delta$-function in the exterior pressure being cancelled by the $\delta$-function in the surface curvature induced by that point pressure.

In general, a discontinuity in slope may be expected to occur at the leading edge, where the free surface (including surface tension) makes contact with the body. On the other hand, smooth separation suggests that no such discontinuity is tolerable at the trailing edge. It is the conclusion of the present paper that a unique solution can be obtained for the linearized planing problem with surface tension, involving both a continuous pressure $P(x)$ over the whole wetted length and an isolated $\delta$-function singularity located at the leading edge. The integral equation corresponding to such a formulation is set up and solved analytically in the long-wave limit, and numerically for some ranges of non-dimensional gravity and surface tension parameters.

Computed results are given here for plane surfaces and for parabolic curved surfaces. In the former case, a non-zero $\delta$-function singularity in the external generating pressure appears to be inevitable, and corresponds (as expected) to a finite jump discontinuity in the slope of the free surface at the point where it converts into the planing surface at its leading edge. The numerical results appear to be well converged, and predict the strength of the $\delta$-function adequately, down to quite small values of the effective surface tension.

However, these results for the flat plate appear to be unacceptable physically. The $\delta$-function strength increases without bound as the surface tension $T$ tends to zero, and a capillary wave is generated ahead of the body, of an amplitude that grows without bound as $T \rightarrow 0$. As the same time, the smooth part of the pressure distribution approaches a limit curve that is quite unlike the known pressure distribution at $T=0$. This paradox is resolved in Part II, which follows.

One can escape this paradox for some families of curved bodies. The results for parabolic planing surfaces indicate that smooth attachment (no leading edge discontinuity of slope), and hence absence of $\delta$-function generating pressure, is possible for suitable choice of the parameters of the parabola. This is equivalent to the process of raising and rotating the basic parabola, described above. The computed results for these special families now have the property that the capillary-wave amplitude tends to zero as $T \rightarrow 0$, and the free-surface shape and pressure distribution now tend to the classical results for $T=0$.

\section{Impulse and step pressures}

The free-surface elevation $y=\eta_{0}(x)$ produced by a $\delta$-function pressure applied on the free surface at $x=0$ is given by Lamb ([3], page 465; see also 
DePrima and $\mathrm{Wu},[2])$, and can readily be computed in terms of the even auxiliary function

$$
f^{\prime}(x)=\cos x \int_{\infty}^{|x|}(\cos t) / t d t+\sin |x| \int_{\infty}^{|x|}(\sin t) / t d t,
$$

of the sine and cosine integrals (Abramowitz and Stegun [1], p. 232, where $f^{\prime}(x)$ is written as $-g(x))$. Thus, if the free stream has speed $U$ in the positive $x$ direction, and $\rho$ is the (constant) fluid density, we have

$$
\rho U^{2} \eta_{0}(x)=K^{\prime}(x)
$$

where

$$
K^{\prime}(x)=-\frac{1}{\pi \nu}\left[f^{\prime}\left(k_{-} x\right)-f^{\prime}\left(k_{+} x\right)\right]-\frac{2}{\nu} \begin{cases}\sin k_{-} x & \text { for } x>0, \\ \sin k_{+} x & \text { for } x<0 .\end{cases}
$$

In (2.3), $k_{-}, k_{+}$are respectively the gravity and capillary wave numbers defined by

$$
k_{ \pm}=\frac{\rho U^{2}}{2 T}(1 \pm \nu),
$$

where (with $g$ the acceleration of gravity and $T$ the surface tension),

$$
\nu=\left(1-\frac{4 g T}{\rho U^{4}}\right)^{1 / 2} .
$$

Figure 1 shows $\frac{1}{2} \rho U^{2} \nu \eta_{0}(x)$, plotted against $k_{-} x$ for various values of $\nu$. A similar plot is given by Lamb. Note that the slope $d \eta_{0} / d x$ is discontinuous at

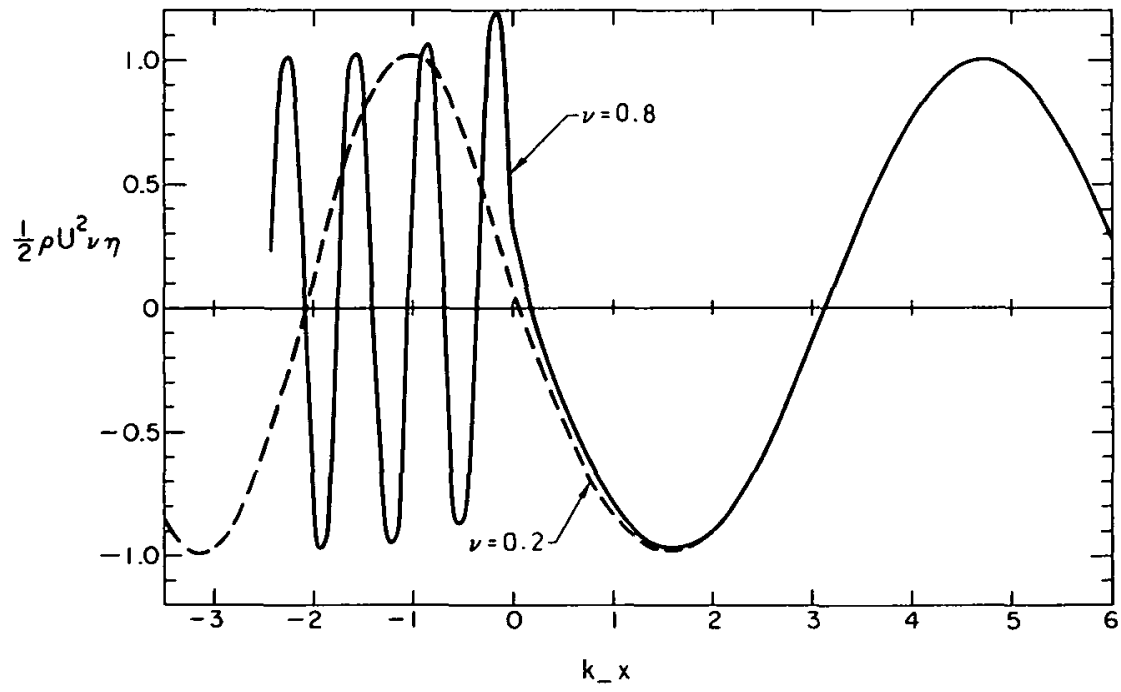

Figure 1. Free-surface shape due to a unit $\delta$-function pressure at $x=0$, for $\nu=0.2(\mu=0.96)$ and $\nu=0.8(\mu=0.36)$. 
$x=0$, but the function $\eta_{0}$ itself is continuous and everywhere bounded, taking a finite value immediately beneath the infinite-pressure point. As $|x| \rightarrow \infty, f^{\prime} \rightarrow 0$, and we obtain gravity waves of wave length $2 \pi / k_{-}$as $x \rightarrow+\infty$ (behind the disturbance) and equal-amplitude (unit) capillary waves of wavelength $2 \pi / k_{+}$ as $x \rightarrow-\infty$, ahead of it.

We are also interested in the free-surface elevation $y=\eta_{1}(x)$ produced by a unit-step pressure, that is, zero pressure for $x<0$ and unit pressure for $x>0$. This is simply the iniegrai of $\eta_{0}$, that is, it satisfies $\eta_{1}^{\prime}(x)=\eta_{0}(x)$, and can be written in terms of the (odd) auxiliary function $f(x)$ whose derivative is $f^{\prime}(x)$ in (2.1). Thus

$$
\rho U^{2} \eta_{1}(x)=K(x)
$$

where

$$
\begin{aligned}
K(x)= & -\frac{1}{\pi \nu}\left[\frac{1}{k_{-}} f\left(k_{-} x\right)-\frac{1}{k_{+}} f\left(k_{+} x\right)\right] \\
& + \begin{cases}\frac{2}{\nu k_{-}} \cos k_{-} x-\frac{1}{\nu}\left(\frac{1}{k_{-}}-\frac{1}{k_{+}}\right) \text {for } x>0, \\
\frac{2}{\nu k_{+}} \cos k_{+} x \text { for } x<0 .\end{cases}
\end{aligned}
$$

Note that, since $f$ also tends to zero at infinity, we obtain capillary waves on $y=0$ as $x \rightarrow-\infty$, but gravity waves on

$$
y=\frac{1}{\rho U^{2}}\left[-\frac{1}{\nu}\left(\frac{1}{k_{-}}-\frac{1}{k_{+}}\right)\right]=-\frac{1}{\rho g}
$$

as $x \rightarrow+\infty$. That is, the waves at $x=+\infty$ are on the equilibrium free surface, depressed hydrostatically by the unit pressure field. The function $\rho g \eta_{1}(x)$ is plotted against $k_{-} x$ in Figure 2 for various $\nu$. Note that the capillary waves are now reduced in amplitude as well as wavelength, compared to the gravity waves, and there is no longer a slope discontinuity at the origin.

In the zero-surface-tension limit $T \rightarrow 0$, we have $\nu \rightarrow 1, k_{+} \rightarrow \infty$ and $k_{-} \rightarrow$ $g / U^{2}$. Thus the elevation due to a step-function pressure is given by

$$
\rho U^{2} \eta_{1}(x) \rightarrow K_{0}(x)
$$

where

$$
\frac{g}{U^{2}} K_{0}(x)=-\frac{1}{\pi} f\left(\frac{g x}{U^{2}}\right)+ \begin{cases}2 \cos \frac{g x}{U^{2}}-1 & \text { for } x>0, \\ 0 & \text { for } x<0,\end{cases}
$$

displaying only pure gravity waves (Lamb [3], page 403). It is also important to note that the degree of singularity at $x=0$ is much increased in this limit, and 


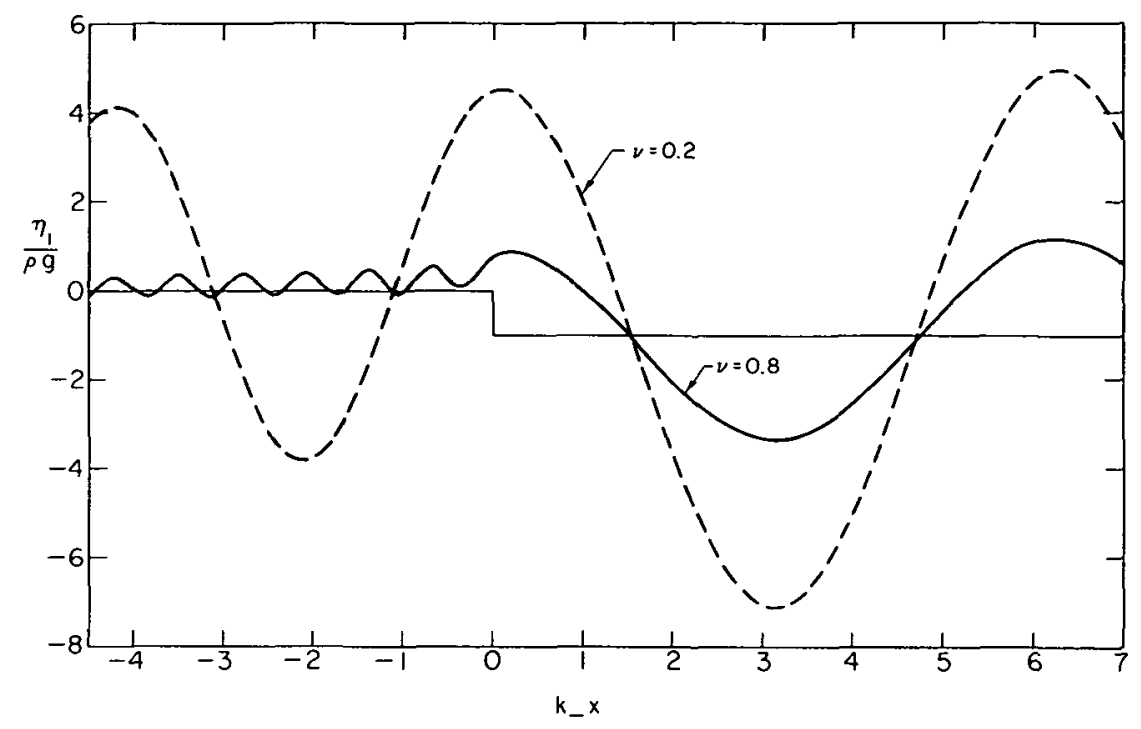

Figure 2. Free-surface shape due to a unit step-function pressure distribution for $\nu=0.2$ and $\nu=0.8$.

we have $\eta_{1}(x)=O(x \log |x|)$ as $x \rightarrow 0$, with infinite slope where the pressure jumps. The situation for the $T \rightarrow 0$ limit of the elevation due to an impulsive pressure is of course even "worse", for then $\eta_{0}(x)=O(\log |x|)$ as $x \rightarrow 0$, is unbounded immediately beneath the pressure point. The expression (2.2) also has the property that the capillary-wave amplitude remains finite as $T \rightarrow 0$, although its wavelength is tending to zero. This is a peculiarity of the $\delta$-function pressure; any smoother forcing (for example, the step function) leads to a vanishing capillary wave as $T \rightarrow 0$. Hence the appropriate limiting expression for $\eta_{0}$ is best thought of as the derivative of $(2.10)$, which has no upstream waves.

\section{The planing integral equation without surface tension}

We now suppose that there is some smooth distribution of pressure

$$
p=\rho U^{2} P(x)
$$

over a portion $|x|<l$ of the free surface. If $T=0$, the resulting free-surface elevation is

$$
\eta(x)=\int_{-l}^{l} d \xi P(\xi) K_{0}^{\prime}(x-\xi)
$$


where

$$
K_{0}^{\prime}(x)=\frac{1}{\pi} f^{\prime}\left(\frac{g x}{U^{2}}\right)- \begin{cases}2 \sin \frac{g x}{U^{2}} & \text { for } \quad x>0, \\ 0 & \text { for } x<0 .\end{cases}
$$

If $\eta(x)$ is given for $|x|<l,(3.2)$ is a weakly-singular Fredholm integral equation to determine $P(x)$. The singularity is logarithmic since, as $x \rightarrow 0$,

$$
K_{0}^{\prime}(x) \rightarrow-(1 / \pi) \log |x|+O(1) .
$$

It is possible to differentiate (3.2) once, to give a Cauchy-principal-value singular integral equation with the slope $\eta^{\prime}(x)$ as input, that is,

$$
\eta^{\prime}(x)=\int_{-l}^{l} d \xi P(\xi) K_{0}^{\prime \prime}(x-\xi),
$$

whose kernel $K_{0}^{\prime \prime}$ possesses a simple-pole singularity at $x=\xi$.

The general theory of such integral equations (for example, Muskhelishvili [4]) indicates that (3.2) possesses a unique solution $P(x)$ for any prescribed $\eta(x)$. In particular, there is a unique solution $P_{0}(x)$ when $\eta(x)=1$, which is also necessarily a solution of the homogeneous case of (3.5), that is, with $\eta^{\prime}(x) \equiv 0$. Thus, (3.5) does not possess a unique solution, its general solution being of the form

$$
P(x)=P_{1}(x)+C P_{0}(x),
$$

where $P_{1}(x)$ is any solution, and $C$ is an arbitrary constant.

An important characteristic of the solution to (3.2) is that in general it is unbounded at both ends $x= \pm l$, possessing inverse-square-root singularities there. In the application to planing surfaces, this is tolerable at the leading edge $x=-l$, where it models spray formation, but intolerble at the trailing edge $x=+l$, where smooth detachment is assumed. In fact, in order to achieve continuity of free-surface slope and planing-surface slope at $x=l$, we must require $P$ to vanish there, that is,

$$
P(l)=0 .
$$

Equation (3.7) is equivalent to the Kutta condition of aerodynamics.

Thus, although (3.2) possesses a unique solution for any given displacement $\eta(x)$, in general that solution is physically unacceptable, since it does not satisfy (3.7). On the other hand, it is always possible to choose the constant $C$ in (3.6) so that (3.7) is satisfied. That is, for any given slope $\eta^{\prime}(x)$, there exists a unique solution of (3.5) satisfying (3.7). Thus, if $\eta_{0}(x)$ is any given displacement, although there may be no acceptable solution for $\eta(x)=\eta_{0}(x)$, there will always be a constant $C$ such that the solution corresponding to

$$
\eta(x)=\eta_{0}(x)+C
$$


does satisfy the Kutta condition (3.7). This means that, if the wetted length $2 l$ is held fixed, we must allow the planing surface to rise or fall bodily, until the Kutta condition is satisfied.

The linearized two-dimensional planing-surface problem in the absence of surface tension, has a long and interesting history, partially surveyed by Wehausen and Laitone in [7], page 587. Good numerical solutions of (3.5) subject to (3.7) are not difficult to achieve, by a number of methods, and some such results are shown in the Figures to follow. The numerical work must be performed at fixed values of the wetted length $2 l$, and is initially parametrized by the Froude number based on that length, or on its reciprocal square, say

$$
\gamma=g l / U^{2} \text {. }
$$

Once the computations are done, it is preferable to re-plot, for example, as done by Squire [6] and Oertel [5], in ways which allow $2 l$ to be determined by the hydrodynamics, as is the case in practice. However, in the present work we retain $\gamma$ as our basic parameter.

\section{The planing integral equation with surface tension}

The equivalent of (3.2) when $T \neq 0$ is

$$
\eta(x)=\int_{-l}^{l} d \xi P(\xi) K^{\prime}(x-\xi),
$$

where $K^{\prime}(x)$ is given by (2.3). This kernel is much less singular than $K_{0}^{\prime}$, and we have, as $x \rightarrow 0$,

$$
K^{\prime}(x) \rightarrow \frac{1}{\pi \nu} \log \frac{k_{+}}{k_{-}}+\left(\frac{k_{+}-k_{-}}{2 \nu}\right)|x|-\left(\frac{k_{+}+k_{-}}{\nu}\right) x+O\left(k_{ \pm}^{2} x^{2} \log |x|\right) .
$$

Although the theory of such non-singular first-kind Fredholm integral equations is much less well developed than the singular case, it is not hard to see by heuristic arguments that we cannot expect any smooth solution of (4.1) for a general input $\eta(x)$ !

For example, the character of the integral equation (4.1) is modelled by the long-wave case $k_{ \pm} \rightarrow 0$, in which the kernel $K^{\prime}$ is replaced by its small- $x$ limit (4.2) everywhere. Then the limiting equation is

$$
\begin{aligned}
\eta(x)= & \int_{-l}^{l}\left[\frac{1}{\pi \nu} \log \frac{k_{+}}{k_{-}}+\frac{k_{+}+k_{-}}{\nu} \xi\right] P(\xi) d \xi-\frac{k_{+}+k_{-}}{\nu} x \int_{-l}^{l} P(\xi) d \xi \\
& +\left(\frac{k_{+}-k_{-}}{2 \nu}\right) \int_{-l}^{l} P(\xi)|x-\xi| d \xi+O\left(k_{ \pm}^{2}\right) .
\end{aligned}
$$


Upon twice differentiating (4.3), we have

$$
P(x)=\eta^{\prime \prime}(x) \nu /\left(k_{+}-k_{-}\right)+O\left(k_{ \pm}^{2}\right),
$$

which appears to determine $P(x)$ uniquely, given only the linearized curvature $\eta^{\prime \prime}$ of the planing surface.

However, the "solution" (4.4) cannot be expected to satisfy (4.3) in general. If we substitite (4.4) intô the rightihand side of (4.3), we must expect to obtain, not $\eta(x)$ itself, but rather $\eta(x)+C-\alpha x$, which involves two unique constants $C$ and $\alpha$, whose effect is lost in the double differentiation. Thus, in order to achieve a smooth pressure distribution $P(x)$ for a given planing surface in the presence of surface tension, we must expect to have to raise or lower the planing surface, and to pitch or rotate it through an angle $\alpha$ that is determined by the hydrodynamics. In particular, for a flat plate $\eta^{\prime}=$ constant, the only smooth pressure distribution is $P(x) \equiv 0$, and $\eta(x) \equiv 0$, that is, no disturbance at all.

The "raise and rotate" procedure can be made the basis of a numerical solution. However, it is clearly too restrictive, and the failure to provide a solution for a flat plate is unacceptable. Why indeed should we expect a "smooth" generating pressure distribution? We have seen in Section 2 that, even when the pressure distribution is as singular as a $\delta$-function, the resulting free-surface elevation is still bounded and continuous. Thus, for example, we may wish to allow $\delta$-function behaviour in $P(x)$. If so, what is the effect on the flow?

As we have seen, a $\delta$-function pressure produces a free-surface slope discontinuity, and hence a weak infinity in the local flow velocity. Such a discontinuity is acceptable at the leading edge, and is inevitable if the planing surface itself possesses internal slope discontinuities, but is apparently unacceptable at the trailing edge. That is, the form taken by the Kutta condition if $T \neq 0$ is no longer (3.7), but simply proscription of $\delta$-function pressure singularities at the trailing edge.

This leaves the leading edge, and internal discontinuities. We consider only leading-edge pressure impulses here; if there are slope discontinuities to the body, the strength of the necessary $\delta$-function is prescribed by that discontinuity, and is not be be considered as an unknown. However, the strength $P_{0}$ of the leading-edge pressure impulse is an unknown, and must be determined as part of the solution process. But, as (4.4) indicates, this is not enough freedom to guarantee a unique solution. We need two disposable constants, and, by analogy with the $T=0$ case, we also allow vertical displacement $C$ of the input planing surface. Thus we suggest, instead of (4.1), the integral equation

$$
\eta(x)+C=P_{0} K^{\prime}(x+l)+\int_{-l}^{l} d \xi P(\xi) K^{\prime}(x-\xi),
$$


where the constants $P_{0}$ and $C$, as well as the smooth pressure distibution $P(x)$, are unknown. We expect, based on the above intuitive arguments, that the system (4.5) will possess a unique solution. Certainly if it does so, the Kutta condition will be satisfied, since $\eta$ will be bounded and continuously differentiable at $x=+l$, and there will be smooth flow separation from the transom.

As an example, suppose we again consider the long-wave (high Froude number) limit $k_{ \pm} \rightarrow 0$. Equation (4.4) still holds and determines the smooth part $P(x)$ of the pressure distribution in terms of the body surface curvature only. In particular, for a flat plate with zero curvature, $P(x) \rightarrow 0$ as $k_{ \pm} \rightarrow 0$. However, this is no longer an unacceptable result, since we still have the term of $P_{0}$ in (4.5) to balance $\eta(x)$. Thus, as $k_{ \pm} \rightarrow 0$, if $\eta^{\prime}(x)=-\alpha=$ constant, we find $P(x) \equiv 0$ but

$$
P_{0}=2 v a /\left(k_{+}+3 k_{-}\right) \text {. }
$$

That is, in this limit, a flat plate is generated simply by a leading-edge $\delta$-function of known strength.

\section{Numerical method}

It is convenient when $T \neq 0$ to differentiate (4.5), thus eliminating the arbitrary constant $C$. We first attempt to solve

$$
\eta^{\prime}(x)=P_{0} K^{\prime \prime}(x+l)+\int_{-l}^{l} d \xi P(\xi) K^{\prime \prime}(x-\xi),
$$

numerically, for given values of the body slope $\eta^{\prime}(x)$. Having done so, we can then compute the unknown vertical shift $C$, simply by computing the position of one point on the body, and comparing this with the input $\eta(x)$.

First we assume that the unknown $P(x)$ is a step function, that is, that $P(x)$ has the form

$$
P(x)=P_{j}, x_{j-1}<x<x_{j} \text { for } j=1,2, \ldots, N,
$$

for some constants $P_{j}, j=1,2, \ldots, N$ to be determined. Thus we have divided the body $(-l, l)$ into $N$ intervals, the $j$ th interval having ends $\left(x_{j-1}, x_{j}\right)$, with $x_{0}=-l, x_{N}=l$, and have approximated the pressure as constant on each interval. Thus

$$
\eta^{\prime}(x)=P_{0} K^{\prime \prime}(x+l)+\sum_{j=1}^{N} P_{j}\left[K^{\prime}\left(x-x_{j-1}\right)-K^{\prime}\left(x-x_{j}\right)\right] .
$$

There are $N+1$ unknowns, $P_{0}, P_{1}, P_{2}, \ldots, P_{N}$; we obtain $N+1$ equations to solve for these unknowns by forcing (5.3) to hold at the $N+1$ end-points 
$x=x_{i}, i=0,1, \ldots, N$. This leads to a set of $N+1$ equations in $N+1$ unknowns, that can be written in vector form as

$$
\mathbf{A p}=\mathbf{b},
$$

where, for all $i=0,1,2, \ldots, N$, the matrix $\mathrm{A}$ has components

$$
A_{i 0}=K^{\prime \prime}\left(x_{i}+l\right) \text {, }
$$

and

$$
A_{i j}=K^{\prime}\left(x_{i}-x_{j-1}\right)-K^{\prime}\left(x_{i}-x_{j}\right) \text { for } j=1,2, \ldots, N \text {, }
$$

with

$$
\mathbf{p}=\left[P_{i}\right] \text { and } \mathbf{b}=\left[\eta^{\prime}\left(x_{i}\right)\right]
$$

In computing the element $A_{00}$, one must be a little careful, since (4.2) indicates that $K^{\prime \prime}(x)$ possesses a jump discontinuity at $x=0$. The correct interpretation of (5.5) for $i=0$ corresponds to the right-hand limit of this step function, since we must force satisfaction of the body boundary condition only for $x$ strictly greater than $-l$. No such problem occurs with $x_{i}=x_{j}$ or $x_{j-1}$ for $j>0$, since $K^{\prime}(x)$ is a continuous function.

The linear system (5.4) may now be solved using any convenient procedure, and yields the pressures and hence forces on the body directly. The matrix $\mathbf{A}$ appears to be well conditioned, and always inverts without difficulty.

The accuracy of the computed results depends only on the number $N$ of intervals of discretization. All computations reported here were performed on a TRS-80 microcomputer which allowed a maximum of 50 intervals. Although a. much finer subdivision would have been possible on a large computer, this value was always sufficient to exhibit the trends in the output.

For any fixed value of $N$, the program loses accuracy when the capillary wavelength becomes comparable to the mean mesh length $2 l / N$. In practice, two or three figure accuracy is attainable with $N=50$, almost down to this wavelength. This is especially so if extrapolation on $N$ is used, exploiting the fact that the error appears to decrease like $N^{-2}$. Although in principle any set of intervals will suffice, a Chebyshev mesh, with

$$
x_{j}=-l \cos (j \pi / N) \text {, }
$$

was found to give best results.

\section{Flat plate solutions}

The program was run first with $\eta^{\prime}(x)=-\alpha=$ constant, representing a flat plate at angle of attack $\alpha$; without loss of generality we may scale $\alpha=1$. Results were computed for various ranges of the parameters, but are presented here only 


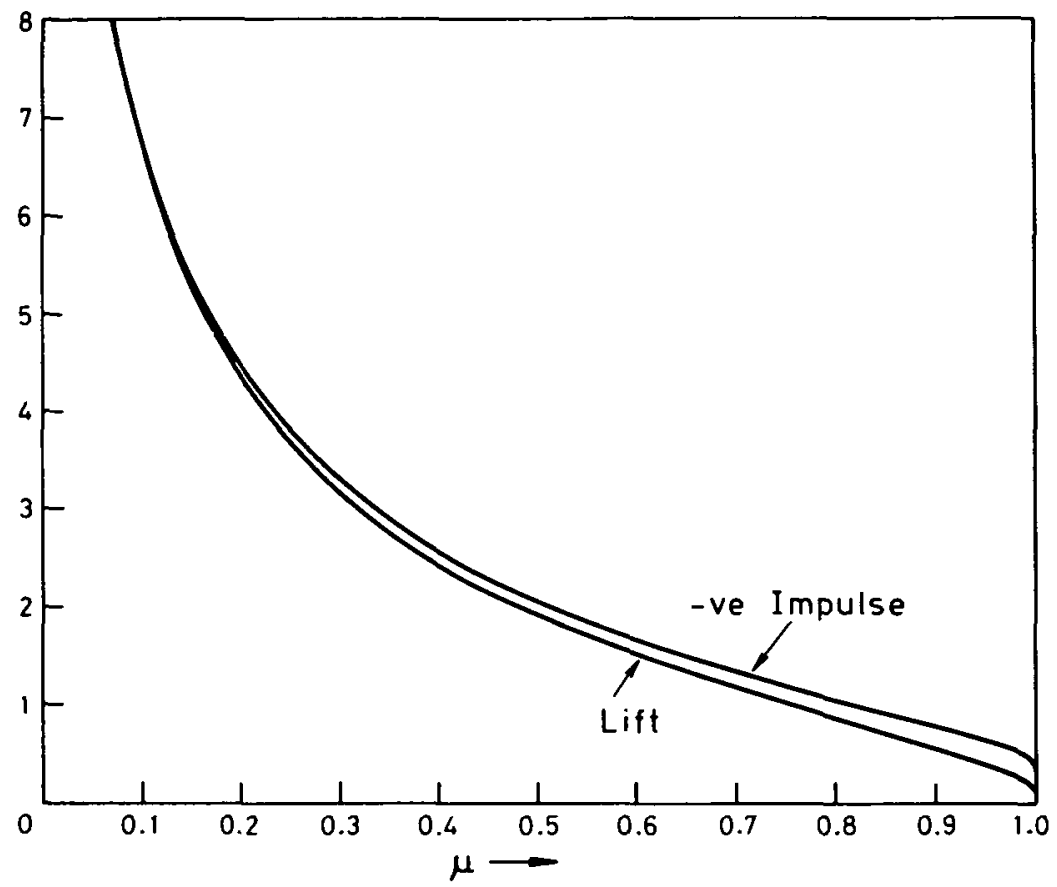

Figure 3. Computed $\delta$-function strength and net lift as a function of scaled surface tension $\mu$, for a flat plate at $\gamma=g l / U^{2}=1$.

for one (typical) Froude number, corresponding to $\gamma=1$. Figure 3 shows a plot of two important output quantities against the non-dimensional surface tension

$$
\mu=4 g T /\left(\rho U^{4}\right)=1-v^{2},
$$

appearing in (2.5). These quantities are the strength $P_{0}$ of the $\delta$-function, and the normalized net lift force

$$
F=\int_{-l}^{l} P(x) d x .
$$

Note that the range of values of $\mu$ is between 0 and 1 . Although $\mu$ can be thought of as a scaled surface tension, $\mu^{-1 / 4}$ can also be thought of as a speed parameter, scaled relative to the minimum speed

$$
U_{m}=[4 g T / \rho]^{1 / 4}
$$

of free gravity-capillary waves. The value of $U_{m}$ is about $23 \mathrm{~cm}$./ $/ \mathrm{sec}$. for air-water interfaces. Thus as $\mu \rightarrow 1, U \rightarrow U_{m}, \nu \rightarrow 0, k_{+} \rightarrow k_{-}$, and the waves ahead and behind the body have the same length. If $\mu>1$, no wave-like flow can exist.

At the other extreme, as $\mu \rightarrow 0$, the effect of surface tension might be expected to vanish. However, there seems no evidence that this is happening here. As 
$\mu \rightarrow 0$, the values of $-P_{0}$ and $F$ both appear to be tending to infinity, while their sum $F+P_{0}$ approaches a constant (negative) value (about -0.13 for $\gamma=1$ ). Although little about this limit is presently understood, it is not in fact surprising that $P_{0}$ tends to minus infinity, in view of the fact [5] that the free surface without surface tension is vertical at its point of contact with the leading edge of the plate. Consideration of the jump in the free-surface slope due to a $\delta$-function pressure (for example, as given by (4.2)) confirms that a vertical slope downward requires a negative-infinite strength to that $\delta$-function.

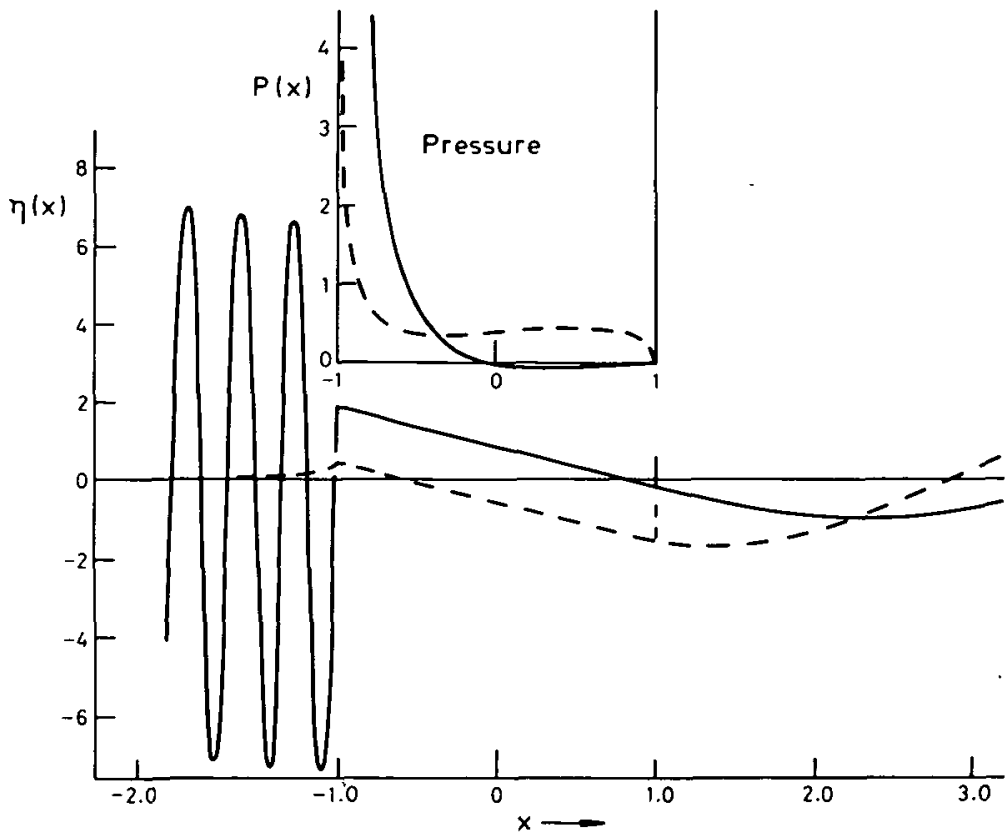

Figure 4. Free-surface shape and pressure distribution for a flat plate at $\gamma=1$ and $\mu=0.2$. Results for zero surface tension shown dashed.

However, the apparent limiting solution as $T \rightarrow 0$ is quite unlike that for $T=0$. Figure 4 shows results, for the case $\mu=0.2$ at $\gamma=1$, for the free-surface shape and the smooth part of the pressure function $P(x)$. At $\mu=0.2$ the capillary wave has a wavelength only $6 \%$ of the gravity wavelength. One might therefore expect quite small effects of surface tension. However, the apparent height of the capillary waves ahead of the plate is rather enormous, some 7 times the vertical displacement between trailing edge and leading edge, and also 7 times the height of the gravity waves behind the plate. The pressure $P(x)$ is concentrated mostly near the leading edge, going negative at about mid-body, but remaining quite small over the whole rear half. 
As $\mu$ decreases below 0.2 , the capillary waves appear to become even higher as they shorten, and the concentration of $P(x)$ near the leading edge increases. In both respects the results are totally dissimilar to the $T=0$ results (for example, those of Oertel [5]) shown dashed on Figure 4. At the same time, there is no comparison between the vertical shifts of the body at $T=0$ and as $T \rightarrow 0$. For example, Figure 4 shows the $\mu=0.2$ output surface only barely touching the equilibrium free surface, with $\eta(1)=-0.2$ but the $T=0$ plate has a substantial draft of about $\eta(1)=-1.6$.

It appears that, at least for a flat plate, the $T=0$ results are not obtainable by taking the continuous limit as $T \rightarrow 0$ of the present results. This paradox is resolved in Part II.

\section{Parabolic surfaces with smooth attachment}

We now consider cases where the input planing surface $\eta=\eta(x)$ is curved, specifically parabolic, with

$$
\eta^{\prime \prime}(x)=\beta=\text { constant }
$$

If, without loss of generality, we set $\eta(0)=0$, and input

$$
\eta(x)=-\alpha x+\frac{1}{2} \beta x^{2},
$$

for some $\alpha$, the program will compute a solution corresponding to $\bar{\eta}(x)=\eta(x)$ $+C$, such that $\bar{\eta}(0)=C$, as in the flat-plate case $\beta=0$. Such a solution, in general, possesses a $\delta$-function singularity at the leading edge, corresponding to free-surface attachment with a finite change in slope, and suffers the same paradox as for the flat-plate case, of failing to approach the $T=0$ solution as $T \rightarrow 0$.

However, among the family of curved bodies, there exists one member (that is, one value of $\alpha$ for every $\beta$ ) such that the attachment is smooth, that is, occurs without change in slope. The solution does not require a leading-edge $\delta$-function, does not generate large-amplitude capillary waves as $T \rightarrow 0$, and may be expected to approach the $T=0$ solution in that limit.

There are two ways to generate this smooth solution. Clearly, we can simply run the standard program, for a range of input values of the angle of attack parameter $\alpha$, and pick out the value of $\alpha$ at which the strength $P_{0}$ of the output $\delta$-function vanishes. Alternatively, we may use the "raise and rotate" procedure described in Section 4, to allow the program to determine $\alpha$ itself. In fact this can be done with only a slight modification of the computational procedure outlined in Section 5. All that is necessary is to replace the term $P_{0} K^{\prime \prime}(x+l)$ in (5.3) by the unknown constant $\alpha$. Hence, the matrix elements $A_{i 0}$ of (5.5) are alt 


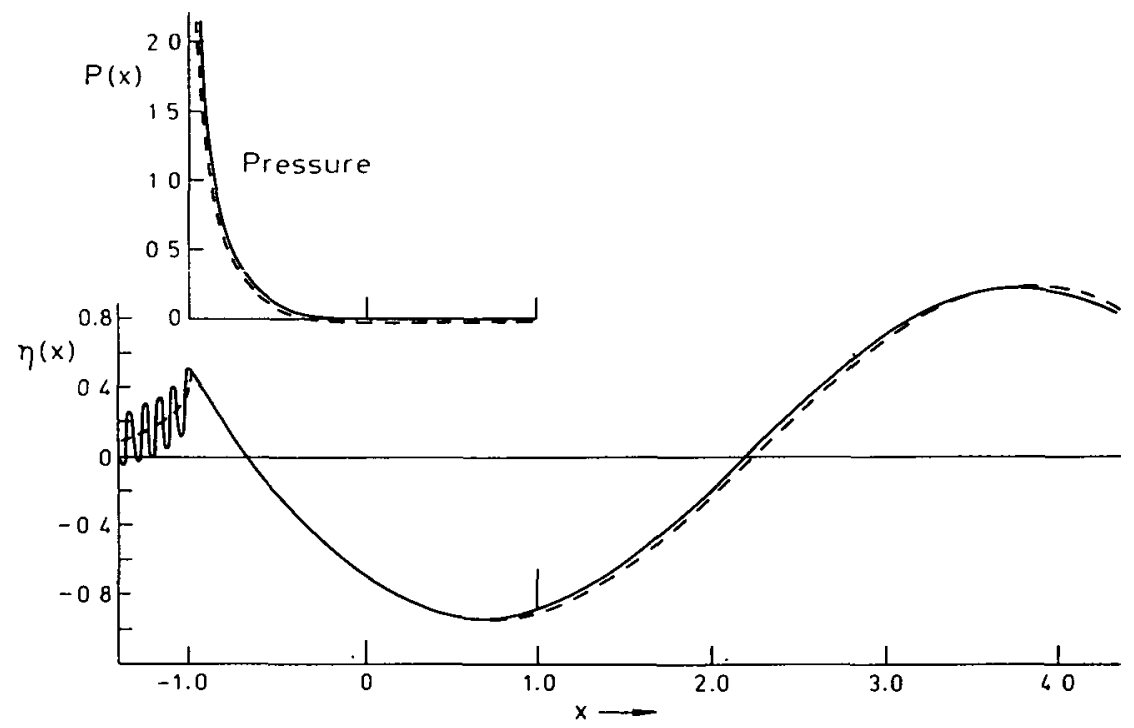

Figure 5. Free-surface shape and pressure distribution for a parabolically curved planing surface at $\gamma=1$ and $\mu=0.05$. Results for zero surface tension shown dashed.

replaced by unity, and the element $i=0$ of the solution vector $\mathrm{p}$ is re-interpreted as $\alpha$, rather than $P_{0}$.

Figure 5 shows the resulting computed free-surface and pressure plots at $\gamma=1$ and $\mu=0.05$, with $\beta$ and $l$ both scaled to unity. The capillary waves are now quite small (about $15 \%$ of the gravity-wave amplitude) and their amplitude is tending to zero as $T \rightarrow 0$. This is shown more clearly by an expanded view of the leading-edge region, in Figure 6 , for $\mu=0.05,0.1$ and 0.2 . This plot shows the (slow) decrease in amplitude as $T \rightarrow 0$, while the wavelength decreases, and also clearly indicates the smooth attachment between free surface and planing surface at the leading edge, for all non-zero values of $T$.

Of course, the planing surface itself is not quite the same, for the three different values of $\mu$ shown in Figure 6, since both the vertical position $C$ and angle of attack $\alpha$ change with $\mu$. However, the difference is less than plotting accuracy in Figure 6. The actual value of $\alpha$ is about 0.7 ; results were also obtained at $T=0$ using Oertel's [5] procedure with $\alpha=0.7$, and showed good agreement with the trend of mean free surface and body surface shapes as $T \rightarrow 0$ in Figure 6.

This is also true of the pressure $P(x)$ shown in Figure 5. It is important to recall that the hydrodynamic pressure felt by the planing surface is not $\rho U^{2} P(x)$ 


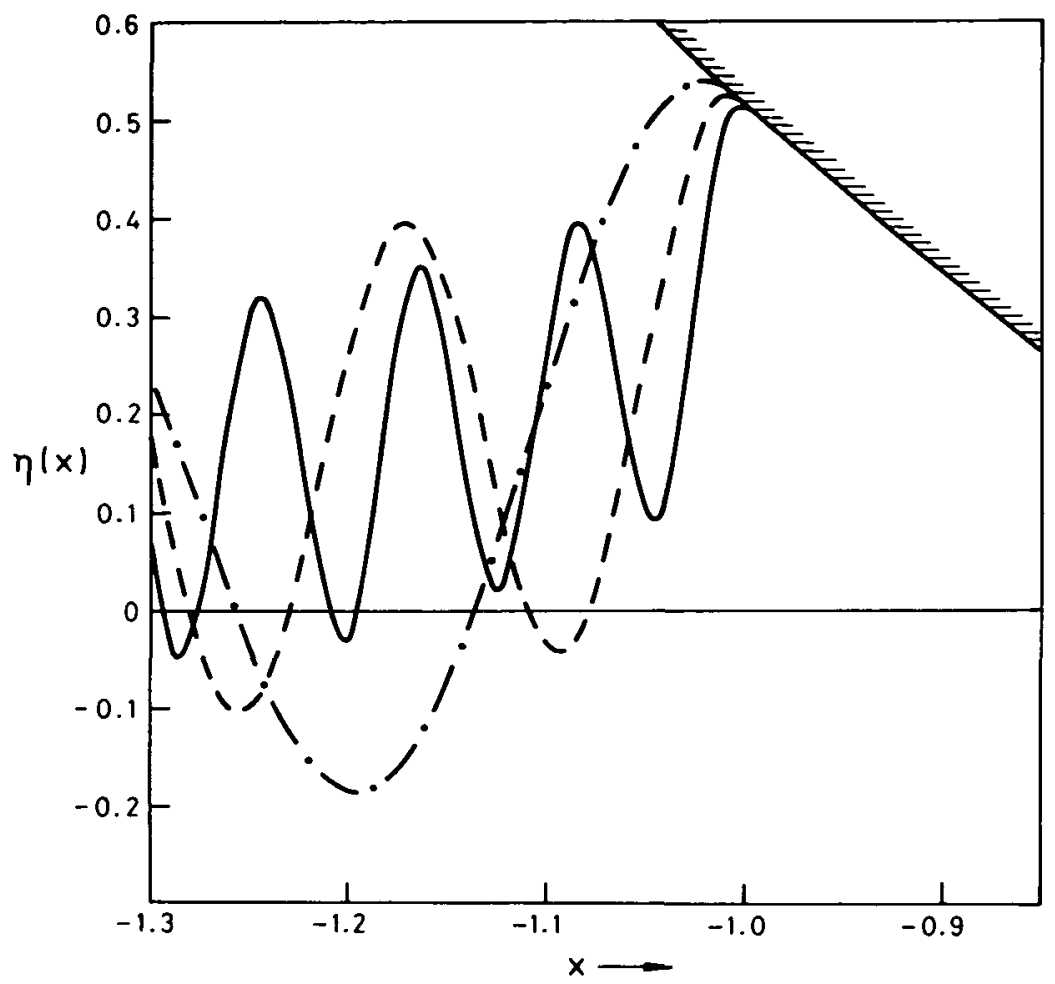

Figure 6. Expanded view of free-surface shape very close to point of contact with leading edge of parabolically-curved planing surface at $\gamma=1$, with $\mu=0.05$ (solid), $\mu=0.1$ (dashed) and $\mu=0.2$ (chain-dotted).

but rather $\rho U^{2} P(x)-T \eta^{\prime \prime}(x)$. However, for the small effective surface tension corresponding to $\mu=0.05$, the correction due to curvature is not significant on the scale of Figure 5 .

\section{References}

[1] M. Abramowitz and I. A. Stegun, Handbook of mathematical functions (Dover, New York, 1964).

[2] C. R. De Prima and T. Y.-T. Wu, "On the theory of surface waves in water generated by moving disturbances," California Institute of Technology, Engineering Division Report 21-23, 1957.

[3] H. Lamb, Hydrodynamics (Cambridge University Press, 1932).

[4] N. I. Muskhelishvili, Singular integral equations (Noordhoff, Groningen, 1953).

[5] R. P. Oertel, The steady motion of a flat ship including an investigation of local flow near the bow (Ph.D. Thesis, University of Adelaide, 1975). 
[6] H. B. Squire, "The motion of a simple wedge along the water surface", Proc. Roy. Soc. A 243 (1957), 48-64.

[7] J. V. Wehausen and E. V. Laitone, "Surface waves," in Handb. der Physik, Vol. 9, ed. S. Flugge (Springer-Verlag, Berlin, 1960).

Department of Applied Mathematics

University of Adelaide

Bux 498, G.P.O.

Adelaide

South Australia 5001 